\title{
Questioning the favorable influence of nerve identification during open inguinal herniorrhaphy is misleading and may increases the risk of surgeons' liability
}

\author{
Invited Commentary: "Does nerve identification during open inguinal herniorrhaphy \\ reduce the risk of persistent pain?"
}

\author{
D. C. Chen · P. K. Amid
}

Received: 22 June 2012/ Accepted: 12 July 2012 / Published online: 31 July 2012

(C) Springer-Verlag 2012

\section{Dear Editors,}

It was with great interest that we read the manuscript "Does nerve identification during open inguinal herniorrhaphy reduce the risk of persistent pain?" The question is both a worthy and challenging one and remains one of the most important issues in inguinal hernia repair. This study's main aim was to correlate intraoperative nerve identification with the risk of nerve damage at 6 months assessed both subjectively and objectively. The authors are to be commended for their work and sound methodology including the use of quantitative sensory testing to help provide some measure of objective data to a complex condition that has primarily been subjectively defined. They report that in their prospective series of 244 patients, despite a high rate of nerve identification, there are still significant signs of nerve damage postoperatively and a risk of persistent pain. Thirty-nine patients $(16.0 \%)$ had substantial pain-related functional impairment at 6-month follow-up. They conclude that "intraoperative nerve identification had no influence on sensory loss in the groin, persistent pain or improvement in functional ability 6 months after surgery."

While the data and conclusions presented are accurate in the context of this series, there are several confounding variables and their conclusions regarding intraoperative nerve identification are not necessarily consistent with current evidence and recommendations for best available

D. C. Chen $(\square)$ · P. K. Amid

Division of General Surgery, Lichtenstein Amid Hernia Clinic at UCLA, David Geffen School of Medicine at UCLA, 1304 15th Street, Suite 213, Santa Monica, CA 90404, USA

e-mail: dcchen@mednet.ucla.edu

P. K. Amid

e-mail: pamid@mednet.ucla.edu practice. The main conclusion that chronic pain and nerve injury persist despite a high rate of nerve identification is limited by a low rate of identification of the genital branch of the genitofemoral nerve. Failure to reliably identify anything less than all three nerves renders the findings inconclusive at best, and it would be misleading to conclude that nerve identification does not decrease rates of pain in routine practice. In order to draw a conclusion that contradicts the best available evidence, the design and data of the study must be supportive. The reported $16 \%$ rate of persistent pain with proper nerve identification is higher than that reported in the literature $[1,2]$. This may be a function of the time point measured, definitions used, low rates of identification of the genital nerve, the use of heavyweight mesh, or improper nerve handling. These variables illustrate the challenges faced by all investigators seeking to define best available technique and practice with regard to the prevention of chronic pain.

It is well known to hernia surgeons that chronic pain has a multifactorial etiology and may develop after hernia operations despite careful handling of nerves and meticulous surgical technique [1, 3-5]. In 2008, the International Consensus Conference was held in Rome with a working group of nine hernia experts and an audience of 200 participants-a group well known to both the authors of this manuscript and the reviewers. This was a culmination of a yearlong review of the best available evidence-based literature with a goal to reach a consensus on several issues related to the prevention and management of chronic pain. While not every answer could be reached with consensus, the issue of nerve identification was well established [1]. Two published studies reporting the results of the role of the identification of all three inguinal nerves $(2,305$ cases all together) with a long follow-up period (ranging from 1 to 5 years) concluded that identification and preservation 
of all three inguinal nerves (this was the case in 924 patients) reduce chronic incapacitating groin pain to less than $1 \%[6,7]$. With proper technique and understanding of groin neuroanatomy, three nerve identification can be readily achieved without increasing the operative time and the incidence of significant chronic pain could be reduced to less than $1 \%[2,3,6,7]$. Based on the best available evidence, the working group and the international audience consensus recommendation was to identify and protect all three inguinal nerves during open inguinal hernia repair to reduce the risk of developing postoperative severe chronic groin pain [1].

The authors report intraoperative nerve identification rates for the iliohypogastric, ilioinguinal, and genitofemoral nerves were $94.7,97.5$, and $21.3 \%$, respectively. In this study, only in 52 of 244 patients had their genital nerve identified, leaving 192 patients without identification. The identification rate of the genital branch $(21.3 \%)$ of the genitofemoral nerve in the current study is lower than that reported in most previous studies addressing this issue. A thorough review of the literature by the International Working Group estimated that in $70-90 \%$ of cases, it is possible to identify all three inguinal nerves [1]. Univariate and multivariate analysis showed that lack of identification of nerves is significantly correlated with presence of chronic pain, the risk of developing inguinal pain increasing with the number of nerves not detected [1]. The authors do acknowledge this but attribute the low rate to definitions and semantics.

In this study, "identification of the genital branch of the genitofemoral nerve was defined as direct verification after necessary dissection, although this is generally avoided as recommended [1]." In the consensus statement, Alfieri et al. do not advocate avoiding direct identification of genital nerve. Rather, dissection of the genital nerve is not routinely needed as the nerve can easily be recognized by the external spermatic vein $[1,2]$. Identification by direct dissection was performed only in case of bleeding of the external spermatic vessels or in case of cremasteric muscle resection and in itself can lead to placing the genital nerve at risk. If nerve identification was performed using this more standard approach, the reported identification rate would likely be higher. We would also clarify the terminology that is interchangeably used in the manuscript that the genitofemoral nerve itself is not in the field of open anterior repair, including the Lichtenstein repair, but rather the genital branch of the genitofemoral nerve.

The $16 \%$ rate of chronic pain (39 of 244 patients) with "substantial pain-related functional impairment at 6-month follow-up" is higher than other studies utilizing nerve identification. This may be attributed to several factors, but recommendations regarding the role of nerve identification and management should be accompanied by comparable outcomes to validate definitions and technique. This higher rate may arise from the definition of pain used by the authors as well as the time point studied. Longer-term results are typically studied for chronic pain, and there may be some difference if the 1- and 5-year time points are evaluated. The authors also report that "a nerve lesion was reported in 5 patients. In 2 of these cases the iliohypogastric nerve were divided, but in the 3 other cases the involved nerves were not specified." Reducing the rate of chronic pain does not come from identification alone, but rather, the information obtained should guide proper management of the nerves. "At-risk" nerves should be resected with proximal intramuscular reimplantation to prevent injury and pain $[1,8]$. Management of the divided iliohypogastric nerves was not specified, but potentially could play a role in the incidence of chronic pain. Additionally, using consensus guidelines, the additional three patients demonstrating a "nerve lesion" would be recommended to have a pragmatic neurectomy of the affected nerve at the initial repair $[1,3,8]$.

The authors state that "a nerve lesion can still occur despite nerve identification, due to entrapment of nerves during mesh fixation and/or mesh inflammation affecting surrounding nerves." This is absolutely true, but both of these causes are associated with improper nerve handling such as inadvertent suturing or fixation and damaging the protective layer of investing fascia covering the nerves, placing them in direct contact with mesh, thus the "mesh inflammation affecting surrounding nerves." This highlights the crucial point that identification of the nerves must be accompanied by proper technique and nerve handling to effectively minimize the rates of pain. The heavyweight meshes that were used in this study are also known to have a higher rate of chronic pain and may contribute to the results. Finally, direct identification of the genital nerve by dissection used in this study may disrupt the protective cremasteric fascia or genital nerve itself and increase the risk of pain [1,2].

The study design is a prospective cohort study, and direct comparison between a group with nerve preservation versus without was not done. Importantly, the authors comment that such a comparison with a "group with no nerve identification would be somewhat unethical based on previous literature." In regard to the citation in the discussion that Reinpold's article of long-term 5-year data of nerve management and rates of chronic pain "suggest[s] that other factors rather than nerve identification are important," the conclusions from that study are not directly comparable [9]. This longitudinal series did not specifically focus on defining the role of nerve identification with the IIN, IHN, and GB identified in only 88.0, 45.3, and $38.2 \%$ of the cases, respectively. Additionally, both Shouldice and Lichtenstein techniques were used, and it was common practice to mobilize the nerve, termed 
"neurolysis" in the article. This series has given some clarity to the issue of nerve mobilization, clearly demonstrating that this should be avoided. The first main conclusion of Reinpold's article is "The nerves should always be visualized, and handled with care [9]."

We hope that the conclusion that "nerve identification had no influence on persistent pain" is not taken out of the context of this study promoting careless nerve handling, leading to patient disability and surgeon liability. As chronic pain has become more salient and the more important outcome measure for inguinal hernia repair, we have seen this issue presented in the mainstream media and this condition has become a common cause for litigation against surgeons. A recent judgment for $\$ 8.2$ million dollars for disability caused by a suture identified on the ilioinguinal nerve is an excessive example of the liability placed upon the surgeon to prevent this injury [10]. We are in complete agreement with the authors that chronic pain is multifactorial and a host of other causes in addition to neuropathy make definition, diagnosis, management, and treatment a challenge [1, 3-5]. However, an emphasis on proper nerve identification, nerve handling, and technique can help to reduce the incidence of this problem. The reported 80-90\% rate of clinical improvement after triple neurectomy demonstrates the important role of nerve injury as a cause of chronic inguinodynia [11]. The authors have also demonstrated similar findings with their experience of selective neurectomy for inguinodynia [12]. Additionally, positive intraoperative findings of nerve injury or entrapment in almost every single case of our nearly 600 groin explorations demonstrate the fallacy of non identification of the nerves and improper nerve handling.

We commend the authors for their ongoing contributions to the understanding of the etiology and prevention of chronic pain. While in agreement that this topic is important and data are crucial to help define best practices, interpreting and generalizing the conclusions of this study may be misperceived. The authors make a concerted effort to emphasize this and "hope that this study serves to stimulate discussion and research on this topic rather than detract from a focus on meticulous nerve identification and proper technique." Despite the complicated results of this study, we are in agreement that it is absolutely imperative for surgeons to understand groin neuroanatomy and practice proper nerve handling. In order to achieve the best possible outcomes for our patients and avoid liability, we recommend identification of all three nerves, making specific mention of the same in the operative report, notation of any variation of the nerves, and resection and documentation of any "nerve at risk." Whether this study answers the question posited by the title requires thoughtful consideration by the readership so that the message and intent is not lost on a simplified answer to a complex question.

\section{References}

1. Alfieri S, Amid PK, Campanelli G, Izard G, Kehlet H, Wijsmuller AR, Di Miceli D, Doglietto GB (2011) International guidelines for prevention and management of post-operative chronic pain following inguinal hernia surgery. Hernia 15(3):239-249

2. Alfieri S, Rotondi F, Di Giorgio A, Fumagalli U, Salzano A, DiMiceli D, Ridolfini MP, Sgagari A, Doglietto G, Group Groin Pain Trial (2006) Groin pain trial group. Influence of preservation versus division of ilioinguinal, iliohypogastric, and genital nerves during open mesh herniorrhaphy: prospective multicentric study of chronic pain. Ann Surg 243(4):553-558

3. Amid PK (2004) Causes, prevention, and surgical treatment of postherniorrhaphy neuropathic inguinodynia: triple neurectomy with proximal end implantation. Hernia 8(4):343-349

4. Amid PK, Hiatt JR (2007) New understanding of the causes and surgical treatment of postherniorrhaphy inguinodynia and orchialgia. J Am Coll Surg 205(2):381-385

5. Amid PK, Chen DC (2011) Surgical treatment of chronic groin pain after laparoscopic and open preperitoneal inguinal hernia repair. J Am Coll Surg 213(4):531-536

6. Izard G, Gailleton R, Randrianasolo S, Houry R (1996) Treatment of inguinal hernia by McVay's technique: a propos of 1332 cases. Ann Chir 50:775-776

7. Lange JF, Wijsmuller AR, van Geldere D, Simons MP, Swart R, Oomen J, Kleinrensink GJ, Jeekel J, Lange JF (2009) Feasibility study of three-nerve-recognizing Lichtenstein procedure for inguinal hernia. Br J Surg 96(10):1210-1214

8. Bartlett DC, Porter C, Kingsnorth AN (2007) A pragmatic approach to cutaneous nerve division during open inguinal hernia repair. Hernia 11(3):243-246

9. Reinpold WM, Nehls J, Eggert A (2011) Nerve management and chronic pain after open inguinal hernia repair: a prospective two phase study. Ann Surg 254(1):163-168

10. Van Heerden $J$ (2008) Inguinal hernia repair and the $\$ 8$ million dollar mistake. Contemp Surg 64(4):183

11. Amid PK (2002) A 1-stage surgical treatment for postherniorrhaphy neuropathic pain: triple neurectomy and proximal end implantation with mobilization of the cord. Arch Surg 137: 100-104

12. Aasvang E, Kehlet H (2009) The effect of mesh removal and selective neurectomy on postherniorrhaphy persistent pain. Ann Surg 171:217-220 